\title{
АППРОКСИМАЦИЯ ЗАДАЧИ ГАРАНТИРОВАННОГО ОЦЕНИВАНИЯ СО СМЕШАННЫМИ ОГРАНИЧЕНИЯМИ ${ }^{1}$
}

\author{
Б. И. Ананьев, П. А. Юровских
}

\begin{abstract}
Рассмотрены вопросы конечномерной аппроксимации задачи гарантированного оценивания для линейных нестационарных систем с возмущениями, подчиненными смешанным интегральным и геометрическим ограничениям. При этом параметры системы и уравнения измерения формируются таким образом, что фазовый вектор системы не подвергается геометрическим ограничениям внутри рассматриваемого отрезка времени. Данные предположения позволяют свести задачу оценивания к задаче оптимального управления без фазовых ограничений и использовать принцип максимума Л. С. Понтрягина. Предложена дискретная многошаговая система, для которой информационное множество сходится в метрике Хаусдорфа к соответствующему информационному множеству непрерывной системы при измельчении разбиения отрезка наблюдения. Получены оценки, характеризующие скорость сходимости. Рассмотрен численный пример для случая раздельных ограничений на начальное состояние и интегральных ограничений на возмущения.
\end{abstract}

Ключевые слова: гарантированное оценивание, фильтрация, вариационные неравенства, нормальный конус, принцип максимума, информационное множество.

B. I. Anan'ev, P. A. Yurovskikh. Approximation of a guaranteed estimation problem with mixed constraints.

Questions of finite-dimensional approximation for a guaranteed estimation problem are considered for linear nonstationary systems with disturbances subject to mixed integral and geometric constraints, where the geometric constraints are not assumed to be compact. The parameters of the system and the measurement equation are formed in such a way that the state vector of the system is not subject to geometric constraints. Under these assumptions, one can reduce the estimation problem to an optimal control problem without state constraints and use Pontryagin's maximum principle. A discrete multistep system is proposed for which the information set converges in the Hausdorff metric to the corresponding information set of a continuous system as the partition step of the observation interval vanishes. Estimates characterizing the convergence rate are derived and an example is given.

Keywords: guaranteed estimation, filtering, variational inequalities, normal cone, maximum principle, information set.

MSC: 93E10, 62L12, 34G25

DOI: $10.21538 / 0134-4889-2020-26-4-48-63$

\section{1. Введение и постановка задачи}

Проблема гарантированного оценивания динамических систем возникла во второй половине прошлого века вскоре после появления теории стохастической фильтрации Р. Калмана и Дж. Бьюси. Основной мотивацией для развития теории гарантированного оценивания послужило отсутствие точных статистических данных о помехах в системе и канале измерения. Одной из первых монографий по гарантированному оцениванию является [1], где существенно развиты и обобщены результаты предыдущих работ [2-4]. Впоследствии идеи упомянутой монографии привели к теории эллипсоидального управления и оценивания (см. [5;6]). Нелинейные системы и численные методы рассмотрены в [7]. Различные вопросы гарантированного оценивания исследовались в [8;9]. Тем не менее работа по созданию устойчивых численных

\footnotetext{
${ }^{1}$ Работа выполнена при поддержке Научно-образовательного центра (НОЦ ИММ УрО РАН), действующего в рамках Уральского математического центра (УМЦ).
} 
методов для гарантированного оценивания еще далека от завершения. В настоящей статье рассматриваем линейную нестационарную систему с наблюдением

$$
\dot{x}(t)=A(t) x(t)+b(t) v(t), \quad y(t)=G(t) x(t)+c v(t), \quad 0 \leqslant t \leqslant T,
$$

где $x(t) \in \mathbb{R}^{n}$ - фазовый вектор, $y(t) \in \mathbb{R}^{m}$ - выход, $v(t) \in \mathbb{R}^{l}$ - неопределенное возмущение; $A(\cdot), G(\cdot), b(\cdot)$ - ограниченные непрерывные матрицы. Предположим, что неопределенные функции $v(\cdot)$ в (1.1) и начальное состояние $x_{0}$ подчинены интегральным и мгновенным ограничениям

$$
\left|x_{0}\right|_{P_{0}}^{2}+\int_{0}^{T}\left(|v(t)|^{2}+2 s^{\prime}(t) x(t)-2 r^{\prime}(t) v(t)\right) d t<1, \quad v(t) \in \mathbf{V}, \quad x_{0} \in X_{0},
$$

где $|\cdot|-$ евклидова норма; $\mathbf{V} \subset \mathbb{R}^{l}$ и $X_{0} \subset \mathbb{R}^{n}-$ выпуклые и замкнутые множества, содержащие нуль; элементы векторов $s(\cdot), r(\cdot)$ принадлежат пространству $L_{2}(0, T)$. Здесь и далее символом $|x|_{P}^{2}$ обозначается квадратичная форма $x^{\prime} P x$, где символ ' означает транспонирование, а матрица $P$ удовлетворяет условию $P^{\prime}=P \geqslant 0$. Кроме того, матрица $c \in \mathbb{R}^{m \times l}$ должна удовлетворять условиям

$$
c c^{\prime}>0, \quad c \mathbf{V}=\mathbb{R}^{m},
$$

первое из которых означает, что $\operatorname{rank} c=m$, а второе будет использоваться для снятия фазовых ограничений внутри рассматриваемого отрезка времени при решении задачи. Ограничения $(1.2),(1.3)$ являются смешанными, т. е. одновременно интегральными и геометрическими. Это один из аспектов новизны в постановке задачи гарантированного оценивания в данной статье. Согласно общей теории гарантированного оценивания, следуя книге [7], дадим определение.

О п р е д е л е н и е. Семейство $\mathcal{X}_{T}(y)=\left\{x_{T}\right\}$ фазовых векторов называется информационным множеством (сокращенно ИМ), если для любого $x_{T} \in \mathcal{X}_{T}(y)$ найдутся функция $v(\cdot)$ и начальное состояние $x_{0}$, удовлетворяющие ограничениям (1.2) и такие, что равенства (1.1) выполняются почти всюду с $x(T)=x_{T}$.

Построить точное множество $\mathcal{X}_{T}(y)$ при ограничениях $(1.2),(1.3)$ - достаточно сложная задача. Поэтому будем решать задачу приближенно. Более конкретно, требуется построить такую систему с дискретным временем и наблюдением, что ее ИМ сходится к $\mathcal{X}_{T}(y)$ в метрике Хаусдорфа при измельчении разбиения отрезка времени. Это требование является вторым аспектом новизны в постановке нашей задачи. Отметим также, что интегральные функционалы, как в (1.2), ранее не рассматривались. K тому же, матрица $P_{0}$ в (1.2) может быть вырожденной или вообще быть равной нулю. Результаты настоящей работы могут использоваться в задачах восстановления входных воздействий и задачах коррекции движения механических систем при наличии коммуникационных ограничений в виде неточных цифровых каналов связи (см. $[9 ; 10])$.

\section{2. Сведение задачи к проблеме оптимального управления}

Обозначим через $C$ матрицу $\left(c c^{\prime}\right)^{-1}$. При условии (1.3) получаем равенства $v(t)=c^{\prime} z(t)+$ $C_{1} v(t)$ и $\|v(\cdot)\|_{T}^{2}=\left\|c^{\prime} z(\cdot)\right\|_{T}^{2}+\left\|C_{1} v(\cdot)\right\|_{T}^{2}$, где $C_{1}=I_{l}-c^{\prime} C c-$ ортогональная проекция на подпространство $\operatorname{ker} c$ и $z(t)=C c v(t)=C(y(t)-G(t) x(t))$. Здесь $\|v(\cdot)\|_{T}^{2}=\int_{0}^{T}|v(t)|^{2} d t$. Используя (1.1), перепишем неравенство в (1.2) как

$$
J\left(T, x_{T}, v, y\right)=\left|x_{0}\right|_{P_{0}}^{2}+\int_{0}^{T}\left(|y(t)-G(t) x(t)|_{C}^{2}+|v(t)|_{C_{1}}^{2}+2 s^{\prime}(t) x(t)\right.
$$




$$
\left.-2 r^{\prime}(t)\left(c^{\prime} C(y(t)-G(t) x(t))+C_{1} v(t)\right)\right) d t<1 .
$$

Нетрудно видеть, что $x_{T} \in \mathcal{X}_{T}(y)$ тогда и только тогда, когда существует функция $v(t) \in \mathbf{V}$, которая удовлетворяет ограничению (2.1) и входит в уравнение

$$
\dot{x}(t)=A(t) x(t)+b(t)\left(C_{1} v(t)+c^{\prime} C(y(t)-G(t) x(t))\right)
$$

с условием $x(T)=x_{T}$ на правом конце. Кроме того, должны выполняться фазовые ограничения

$$
y(t)-G(t) x(t) \in c \mathbf{V}, \quad x_{0} \in X_{0},
$$

но первое из них снимается ввиду предположения (1.3). С другой стороны, такая функция существует тогда и только тогда, когда минимум левой части неравенства $(2.1)$ по $v(\cdot)$ согласно уравнению (2.2) при фазовых ограничениях (2.3) будет меньше единицы. При этом определится некоторый вектор $x(T)=x_{T}$ на правом конце траектории. Введем обозначения. Пусть

$$
\tilde{b}(t)=b(t) c^{\prime} C, \quad \widetilde{A}(t)=A(t)-\tilde{b}(t) G(t) .
$$

Тогда можем записать

$$
\begin{gathered}
\dot{x}(t)=\widetilde{A}(t) x(t)+\tilde{b}(t) y(t)+b(t) C_{1} v(t), \\
x(t)=\widetilde{X}(t, T) x_{T}-\int_{t}^{T} \widetilde{X}(t, s)\left(\tilde{b}(s) y(s)+b(s) C_{1} v(s)\right) d s
\end{gathered}
$$

вместо $(2.2)$, где $\widetilde{X}(t, s)$ - фундаментальная матрица уравнения $\dot{x}(t)=\widetilde{A}(t) x(t)$. Итак, справедливо следующее утверждение.

Лемма. Вектор $x_{T} \in \mathcal{X}_{T}(y)$ тогда и толъко тогда, когда минимум функиионала $J(T$, $\left.x_{T}, v, y\right)$ в (2.1) по $v(\cdot)$ при условиях дифференииальной связи (2.5) и фазовых ограничениях (2.3) меньше единицы.

Отметим, что условие (1.3) будет выполнено, если, например, $\mathbf{V}=\mathbf{W} \times \mathbb{R}^{m}, \mathbf{W} \subset \mathbb{R}^{k}-$ выпуклое и замкнутое множество, $v(t)=[w(t) ; \xi(t)], l=k+m$. Матрицы в $(2.1)$ в этом случае имеют вид

$$
b(t)=\left[B(t), O_{n \times m}\right], \quad c=\left[O_{m \times k}, I_{m}\right] .
$$

Здесь $O_{n \times m} \in \mathbb{R}^{n \times m}$ - нулевая матрица. В случае (2.6) имеем $C=I_{m}$ (единичная матрица размера $m \times m)$, величина $\tilde{b}(t)=0$ в $(2.4)$. Неравенство (2.1) примет вид

$$
\begin{aligned}
& J\left(T, x_{T}, v, y\right)=\left|x_{0}\right|_{P_{0}}^{2}+\int_{0}^{T}\left(|y(t)-G(t) x(t)|^{2}+|w(t)|^{2}+2 s^{\prime}(t) x(t)\right. \\
& \left.-2 r_{1}^{\prime}(t) w(t)-2 r_{2}^{\prime}(t)(y(t)-G(t) x(t))\right) d t<1, \quad r(t)=\left[r_{1}(t) ; r_{2}(t)\right] .
\end{aligned}
$$

Уравнения (2.5) заменятся на следующие:

$$
\dot{x}(t)=A(t) x(t)+B(t) w(t), \quad x(t)=X(t, T) x_{T}-\int_{t}^{T} X(t, s) B(s) w(s) d s .
$$

В соотношениях (2.6)-(2.8) и далее используются обозначения $[A, B],[A ; B]$ для горизонтальной и вертикальной конкатенации, соответственно, матриц и векторов. Обозначение $[A, B ; C, D]$ - это блочная матрица вида $\left[\begin{array}{l}A B \\ C D\end{array}\right]$. При этом размерности матриц и векторов должны быть согласованы. 


\section{3. Задача без геометрических ограничений}

Пусть $\mathbf{V}=\mathbb{R}^{l}$ и $X_{0}=\mathbb{R}^{n}$, т. е. геометрические ограничения отсутствуют. Этот случай подробно исследуется в данном разделе, поскольку результаты со структурой интегрального функционала в (1.2), (2.1) существенно используются для построения дискретной многошаговой аппроксимации. Также совместные квадратичные ограничения применяются для аппроксимации задач с раздельными ограничениями. Используем принцип максимума (см. [11, теорема 1, с. 437]). Минимизируем функционал в левой части неравенства (2.1) при закрепленном правом конце траектории системы (2.5). Имеем выражение для гамильтониана

$$
\begin{gathered}
H\left(t, x, v, \psi, a_{0}\right)=-a_{0}\left(|y(t)-G(t) x|_{C}^{2}+|v|_{C_{1}}^{2}+2 s^{\prime}(t) x-2 r^{\prime}(t)\left(c^{\prime} C(y(t)-G(t) x)+C_{1} v\right)\right) \\
+\psi^{\prime}\left(\widetilde{A}(t) x+\tilde{b}(t) y(t)+b(t) C_{1} v\right) .
\end{gathered}
$$

Сопряженная система запишется как

$$
\dot{\psi}(t)=-2 a_{0}\left(G^{\prime}(t) C(y(t)-G(t) x(t)-c r(t))-s(t)\right)-\widetilde{A}^{\prime}(t) \psi(t), \quad 0 \leqslant t \leqslant T .
$$

Условие на левом конце $\psi(0)=2 a_{0} P_{0} x_{0}$. Условие на правом конце для системы (3.1) смысла не имеет. В силу однородности и линейности функций в $H$ и (3.1) можно положить $a_{0}=1 / 2$. Условие максимума с $a_{0}=1 / 2$ в нашем случае приводит к уравнению

$$
C_{1}\left(b^{\prime}(t) \psi(t)+r(t)-v(t)\right)=0 .
$$

Условие (3.2), в котором слева стоит градиент функции $H\left(t, x, v, \psi, a_{0}\right)$ по $v$, должно выполняться почти всюду на рассматриваемом отрезке времени. Следует иметь в виду, что для линейно выпуклых задач принцип максимума является как необходимым, так и достаточным условием оптимальности. Таким образом, получаем двухточечную краевую задачу для уравнения (2.5) и сопряженного уравнения

$$
\dot{\psi}(t)=-\widetilde{A}^{\prime}(t) \psi(t)-G^{\prime}(t) C(y(t)-G(t) x(t)-c r(t))+s(t), \quad \psi(0)=P_{0} x_{0},
$$

с поточечным условием (3.2). Данная система решается подстановкой $\psi(t)=P(t) x(t)+p(t)$. Здесь для $P(t)$ получается матричное уравнение Риккати с начальным условием $P(0)=P_{0}$, а для $p(t)$ - векторное уравнение с нулевым начальным условием. Действительно, имеем следующие уравнения:

$$
\begin{gathered}
\dot{P}(t)=-P(t) \widetilde{A}(t)-\widetilde{A}^{\prime}(t) P(t)+G^{\prime}(t) C G(t)-P(t) b(t) C_{1} b^{\prime}(t) P(t), \quad P(0)=P_{0} ; \\
\dot{p}(t)=-\widetilde{A}(t) p(t)-P(t)\left(\tilde{b}(t) y(t)+b(t) C_{1}\left(b^{\prime}(t) p(t)+r(t)\right)\right)-G^{\prime}(t) C(y(t)-c r(t))+s(t),
\end{gathered}
$$

где $p(0)=0$. Из $(3.2)$ находим оптимальное возмущение с обратной связью

$$
C_{1} v(t)=C_{1}\left(b^{\prime}(t)(P(t) x(t)+p(t))+r(t)\right) .
$$

Если подставить это возмущение в уравнение (2.5) и выписать его решение

$$
x(t)=\bar{X}(t, T) x_{T}-\int_{t}^{T} \bar{X}(t, s)\left(\tilde{b}(s) y(s)+b(s) C_{1}\left(b^{\prime}(s) p(s)+r(s)\right)\right) d s,
$$

где $\bar{X}(t, s)$ - фундаментальная матрица уравнения $\dot{x}(t)=\left(\widetilde{A}(t)+b(t) C_{1} b^{\prime}(t) P(t)\right) x(t)$, то после подстановки решения в неравенство (2.1) вместе с оптимальным возмущением (3.4) получаем соотношение

$$
x_{T}^{\prime} P(T) x_{T}-2 x_{T}^{\prime} d(T)+e(T)<1,
$$


задающее уравнение эллипсоида для правых концов траектории и определяющее ИМ $\mathcal{X}_{T}(y)$. Здесь имеет место равенство матриц

$$
P(T)=\int_{0}^{T} \bar{X}^{\prime}(t, T)\left(G^{\prime}(t) C G(t)+P(t) b(t) C_{1} b^{\prime}(t) P(t)\right) \bar{X}(t, T) d t+\bar{X}^{\prime}(0, T) P_{0} \bar{X}(0, T),
$$

в чем можно убедиться путем дифференцирования по параметру $T$. Для невырожденности эллипсоида $\mathcal{X}_{T}(y)$ для любого $T>0$ достаточно потребовать полной наблюдаемости системы $(2.5)$ на любом отрезке $0 \leqslant t \leqslant T$. Последнее означает положительную определенность матрицы

$$
\int_{0}^{T} \tilde{X}^{\prime}(t, T) G^{\prime}(t) G(t) \tilde{X}(t, T) d t>0
$$

что влечет невырожденность матрицы (3.6). Для параметров $d$, e также выписываются дифференциальные уравнения, которые следуют из формул (2.1), (3.3), (3.4), (3.6). Однако проще их получить с помощью динамического программирования. Для этого запишем уравнение Беллмана для функции $V(t, x)=\min _{v(\cdot)} J\left(t, x_{t}, v, y\right)$ в задаче минимизации, в которой левый конец свободен, а правый фиксирован: $x(t)=x_{t}$. Имеем

$$
\begin{gathered}
V_{t}=\min _{v}\left\{-\left(\widetilde{A}(t) x+\tilde{b}(t) y(t)+b(t) C_{1} v\right)^{\prime} V_{x}+|y(t)-G(t) x|_{C}^{2}+|v|_{C_{1}}^{2}+2 s^{\prime}(t) x\right. \\
\left.-2 r^{\prime}(t)\left(c^{\prime} C(y(t)-G(t) x)+C_{1} v\right)\right\} .
\end{gathered}
$$

Минимум здесь достигается на возмущении $C_{1} v=C_{1}\left(r(t)+b^{\prime}(t) V_{x} / 2\right)$. Решение для уравнения (3.8) находится в виде квадратичной формы вида (3.5), где для $P(t)$ выполняется первое уравнение в (3.3). Для параметров $d, e$ получаются уравнения

$$
\begin{gathered}
\dot{d}(t)=P(t) \tilde{b}(t) y(t)-\widetilde{A}^{\prime}(t) d(t)+G^{\prime}(t) C(y(t)-c r(t))+P(t) b(t) C_{1}\left(r(t)-b^{\prime}(t) d(t)\right)-s(t), \\
\dot{e}(t)=2 y^{\prime}(t) \tilde{b}^{\prime}(t) d(t)+|y(t)|_{C}^{2}-2 r^{\prime}(t) c^{\prime} C y(t)-\left|r(t)-b^{\prime}(t) d(t)\right|_{C_{1}}^{2}
\end{gathered}
$$

с нулевыми начальными условиями $d(0)=e(0)=0$.

В случае невырожденности (3.7) или условии $P_{0}>0$ неравенство (3.5) переписывается в виде

$$
\left|x_{T}-\hat{x}(T)\right|_{P(T)}^{2}+h(T)<1,
$$

где параметры $\hat{x}, h$ удовлетворяют дифференциальным уравнениям

$$
\begin{gathered}
\dot{\hat{x}}(t)=A(t) \hat{x}(t)+\left(b(t) c^{\prime} C+P^{-1}(t) G^{\prime}(t) C\right)(y(t)-G(t) \hat{x}(t)-c r(t)) \\
-P^{-1}(t) s(t)+b(t) r(t), \quad \dot{h}(t)=|y(t)-G(t) \hat{x}(t)-c r(t)|_{C}^{2}-|r(t)|^{2}+2 s^{\prime}(t) \hat{x}(t) .
\end{gathered}
$$

Однако найти величины $\hat{x}(T), h(T)$ в (3.10) из этих уравнений нельзя, поскольку начальные условия, вообще говоря, не определены. Но они будут определенными и нулевыми, если

$$
P_{0}^{\prime}=P_{0}>0 \text {. }
$$

В этом можно убедиться, переходя к пределу $t \rightarrow 0$ в соотношениях $\hat{x}(t)=P^{-1}(t) d(t)$ и $h(t)=e(t)-d^{\prime}(t) P^{-1}(t) d(t)$. Условие невырожденности (3.7) тогда становится излишним. Таким образом, справедлива следующая теорема. 
Теорема 1. При отсутствии геометрических ограничений и наличии интегральных ограничений (1.2), (2.1) ИМ $\mathcal{X}_{T}(y)$ представляет собой эллипсоид, определяемый неравенством (3.5). Параметры эллипсоида находятся путем решения дифберенииальных уравнений (3.3) и (3.9). В случае невырожсденности (3.7) величины $\hat{x}(t), h(t)$ из представления ИМ в виде (3.10) удовлетворяют уравнениям (3.11). При условии (3.12) эллипсоид является невырожденным и без неравенства (3.7), причем уравнения (3.11) решаются с нулевыми начальными условиями.

Отметим, что в случае $s(t)=r(t)=0$ уравнения (3.11) сводятся к ранее известным (см. [1]).

\section{4. Задача при геометрических и интегральных ограничениях на возмущения}

Предположим, что заданы ограничения (1.2). Условия (1.3) считаются выполненными. Рассмотрим индикаторную функцию произвольного множества $X$, определяемую формулой

$$
\psi_{X}(x)= \begin{cases}0, & \text { если } x \in X ; \\ +\infty, & \text { если } x \notin X .\end{cases}
$$

Ограничения (2.1) можем записать как

$$
\psi_{X_{0}}\left(x_{0}\right)+J\left(T, x_{T}, v, y\right)<1, \quad v(t) \in \mathbf{V} .
$$

Для определения ИМ $\mathcal{X}_{T}(y)$ требуется минимизировать функционал в левой части неравенства (4.1) в силу уравнения из (2.5). Классический вариант принципа максимума здесь не проходит, поскольку функционал содержит бесконечные значения. Однако имеются обобщения (см. [12]), в которых производные заменяются субдифференциалами, а многие равенства включениями. В нашем случае задача остается выпуклой, поэтому необходимые условия оптимальности, по-прежнему, будут и достаточными.

Введем в рассмотрение следующие множества:

$N_{\mathbf{V}}(z)=\left\{v \in \mathbb{R}^{l} \mid v^{\prime}(x-z) \leqslant 0, \forall x \in \mathbf{V}\right\}$ - выпуклый нормальный конус к замкнутому выпуклому множеству $\mathbf{V} \subset \mathbb{R}^{l}$ в точке $z$ и

$N_{X_{0}}(x)=\left\{g \in \mathbb{R}^{n} \mid g^{\prime}(w-x) \leqslant 0, \forall w \in X_{0}\right\}$ - выпуклый нормальный конус к замкнутому выпуклому множеству $X_{0} \subset \mathbb{R}^{n}$ в точке $x$.

Известно, что для дифференцируемой выпуклой функции $f(x)$ многих переменных субдифференциал $\partial f(x)$ совпадает с градиентом $\nabla f(x)$ и субдифференциал суммы функций равен сумме субдифференциалов. Для индикаторной выпуклой функции имеем равенство $\partial \psi_{X}(x)=N_{X}(x)$.

Применяя обобщенный принцип максимума (см. [12]) и используя [13, теорема 6Н.1], получаем краевую задачу и условие трансверсальности (здесь $\left.a_{0}=1 / 2\right)$ на левом конце траектории:

$$
\begin{gathered}
\dot{x}(t)=\widetilde{A}(t) x(t)+\tilde{b}(t) y(t)+b(t) C_{1} v(t), \quad x(T)=x_{T}, \\
\dot{\psi}(t)=-\widetilde{A}^{\prime}(t) \psi(t)-G^{\prime}(t) C(y(t)-G(t) x(t)-c r(t))+s(t), \quad \psi(0) \in P_{0} x_{0}+N_{X_{0}}\left(x_{0}\right) .
\end{gathered}
$$

При этом должно выполняться необходимое и достаточное условие максимума

$$
C_{1}\left(b^{\prime}(t) \psi(t)+r(t)-v(t)\right) \in N_{\mathbf{V}}(v(t)) \text { для почти всех } t, \quad 0 \leqslant t \leqslant T,
$$

для оптимальной функции $v(\cdot)$.

Свойства оптимального возмущения задачи в функциональных пространствах. Представим задачу в гильбертовом пространстве $L_{2}$. Параметры $T$ и $x_{T}$ зафиксируем. Решение 
уравнения в (2.5) запишем в операторном виде как

$$
\begin{gathered}
x=\mathcal{T} b C_{1} v+\mathcal{W}(y), \quad \mathcal{T} w=-\int_{t}^{T} \tilde{X}(t, s) w(s) d s, \\
\mathcal{W}(y)=\tilde{X}(t, T) x_{T}-\int_{t}^{T} \tilde{X}(t, s) \tilde{b}(s) y(s) d s,
\end{gathered}
$$

где $\mathcal{T}: L_{2}^{n} \rightarrow L_{2}^{n}$ и $\mathcal{W}: L_{2}^{m} \rightarrow L_{2}^{n}-$ непрерывные операторы, причем $\mathcal{T}$ - линейный, а $\mathcal{W}-$ аффинный. Поскольку отрезок времени фиксирован, то он не упоминается в обозначении пространств $L_{2}$ разной размерности. Аналогично имеем представление $x_{0}=\mathcal{T}_{0} b C_{1} v+\mathcal{W}_{0}(y)$, где $\mathcal{T}_{0}: L_{2}^{n} \rightarrow \mathbb{R}^{n}$ и $\mathcal{W}_{0}: L_{2}^{m} \rightarrow \mathbb{R}^{n}-$ непрерывные операторы. Введем скалярное произведение $\langle\cdot, \cdot\rangle$ в пространстве $L_{2}^{l}$. Тогда функционал для минимизации можно представить как

$$
\langle v, \mathcal{A} v\rangle-2\langle v, \mathcal{V}(\mathbf{y})\rangle
$$

где $\mathcal{A}=C_{1}+C_{1} b^{*}\left(\mathcal{T}_{0}^{*} P_{0} \mathcal{T}_{0}+\mathcal{T}^{*} G^{*} C G \mathcal{T}\right) b C_{1}$ - однородный оператор, оператор $\mathcal{V}$ зависит от параметра $\mathbf{y}=(y, r, s) \in L_{2}^{m+l+n}$ и определяется по формуле

$$
\mathcal{V}(\mathbf{y})=C_{1} b^{*}\left(-\mathcal{T}_{0}^{*} P_{0} \mathcal{W}_{0}(y)+\mathcal{T}^{*}\left(G^{*} C(y-G \mathcal{W}(y)-c r)-s\right)\right)+C_{1} r
$$

В функционале (4.5) опущены слагаемые, не зависящие от $v$. Отметим следующее. В формулах присутствует симметричный проективный оператор $C_{1}$ на линейное подпространство $\operatorname{ker} c$. Поэтому можно считать, что $v \in L_{2}((0, T), \operatorname{ker} c)$. Рассмотрим множество

$$
\begin{gathered}
\mathbb{V}(y)=\left\{v \in L_{2}^{l} \mid v(t) \in \mathbf{V} \text { для почти всех } t, 0 \leqslant t \leqslant T\right\} \\
\bigcap\left\{v \in L_{2}^{l} \mid \mathcal{T}_{0} b C_{1} v+\mathcal{W}_{0}(y) \in X_{0}\right\} .
\end{gathered}
$$

Прилагая [13, теорема 6Н.1], приходим к необходимому и достаточному условию оптимальности

$$
\mathcal{V}(\mathbf{y})-\mathcal{A} v \in N_{\mathbb{V}(y)}(v),
$$

где $N_{\mathbb{V}(y)}(v)$ - выпуклый нормальный конус в точке $v$ к выпуклому и замкнутому множеству (4.6) в пространстве $L_{2}^{l}$. При этом оператор $\mathcal{A}$ в данном пространстве удовлетворяет неравенству $\langle v, \mathcal{A} v\rangle \geqslant\left\langle v, C_{1} v\right\rangle$, т. е. является коэрцитивным с параметром единица на $L_{2}((0, T), \operatorname{ker} c)$.

В вариационном неравенстве (4.7) величина у служит параметром. В силу строгой монотонности величины $\mathcal{V}(\mathbf{y})-\mathcal{A} v$ по $v$ для всякого у существует единственное решение $v(\mathbf{y}) \in$ $L_{2}((0, T), \operatorname{ker} c)$ этого неравенства, которое к тому же будет глобально липшицевым по паpe $(r, s)$. Действительно, пусть $\mathbf{y}_{1}$ и $\mathbf{y}_{2}$ - два различных параметра с одинаковым сигналом $y$, но с разными $(r, s)$. Тогда $\left\|v\left(\mathbf{y}_{1}\right)-v\left(\mathbf{y}_{2}\right)\right\|_{C_{1}} \leqslant\left\|\mathcal{V}\left(\mathbf{y}_{1}\right)-\mathcal{V}\left(\mathbf{y}_{2}\right)\right\|_{C_{1}}$ по [13, теорема 2F.6]. Здесь символ $\|w\|_{C_{1}}$ означает $\sqrt{\left\langle w, C_{1} w\right\rangle}$. Разность $\mathcal{V}\left(\mathbf{y}_{1}\right)-\mathcal{V}\left(\mathbf{y}_{2}\right)$ представляет собой линейный однородный оператор относительно разности $\mathbf{y}_{1}-\mathbf{y}_{2}$. Следовательно, получаем необходимое неравенство $\left\|v\left(\mathbf{y}_{1}\right)-v\left(\mathbf{y}_{2}\right)\right\|_{C_{1}} \leqslant \alpha\left\|\mathbf{y}_{1}-\mathbf{y}_{2}\right\|$ для некоторой нормы в произведении пространств $L_{2}^{m+l+n}$ и некоторой константы $\alpha>0$. Полученная оценка равномерна по одинаковым сигналам $y$ в параметрах $\mathbf{y}_{1}$ и $\mathbf{y}_{2}$. Сформулируем теорему.

Теорема 2. Определим отображение $S$, сопоставляющее параметру у оптимальную пару $(v(\cdot), x(\cdot)) \in L_{2}^{l} \times \mathcal{C}^{n}$ согласно (4.7), где $\mathcal{C}^{n}-$ пространство непрерывных $n$-векторных функиий при $0 \leqslant t \leqslant T$. Тогда отображение $S: L_{2}^{l+n} \rightarrow L_{2}^{l} \times \mathcal{C}^{n}$ оказывается глобально липиицевым nо $(r, s)$ равномерно по всем сигналам $y$. 
Д о к а з а т е л ь с т в о. Свойство липшицевости оптимальной функции $v(\mathbf{y})(\cdot)$ по $(r, s)$ уже установлено. Проверим это свойство для $x(\mathbf{y})$. Обозначим функцию $x\left(\mathbf{y}_{i}\right)(t)$ через $x_{i}(t)$ для $i=1,2$. Из формулы (4.4) получаем

$$
\begin{gathered}
\left|x_{1}(t)-x_{2}(t)\right| \leqslant \int_{t}^{T}|\widetilde{X}(t, s)|\left(|b(s)|\left|C_{1}\left(v\left(\mathbf{y}_{1}\right)(s)-v\left(\mathbf{y}_{2}\right)(s)\right)\right|+|\tilde{b}(s)|\left|y_{1}(s)-y_{2}(s)\right|\right) d s \\
\leqslant \alpha_{1}\left\|v\left(\mathbf{y}_{1}\right)-v\left(\mathbf{y}_{2}\right)\right\|_{C_{1}}+\alpha_{2}\left\|y_{1}-y_{2}\right\| \leqslant\left(\alpha_{1} \alpha+\alpha_{2}\right)\left\|\mathbf{y}_{1}-\mathbf{y}_{2}\right\| .
\end{gathered}
$$

Здесь $\left\|y_{1}-y_{2}\right\|=0$, тем не менее мы оставляем эти разности в оценке для формулировки замечания после теоремы. Вычисляя в полученной формуле максимум по $t$, получаем требуемое.

3 а м е ч а н и е 1 . Пусть $X_{0}=\mathbb{R}^{n}$. Тогда из рассуждений теоремы 2 следует, что отображение $S: L_{2}^{m+l+n} \rightarrow L_{2}^{l} \times \mathcal{C}^{n}$ оказывается глобально липшицевым по $\mathbf{y}$.

Введем сопряженный элемент $\psi$ по формуле

$$
\psi=-\mathcal{T}_{0}^{*} P_{0} x_{0}+\mathcal{T}^{*}\left(G^{*} C(y-G x-c r)-s\right),
$$

где $x$ определяется по формуле (4.4). Тогда, вычисляя сопряженные операторы, нетрудно проверить, что $\psi(\cdot)$ представляет собой абсолютно непрерывную функцию, удовлетворяющую второму уравнению в (4.2) и условию трасверсальности $\psi(0) \in P_{0} x_{0}+N_{X_{0}}\left(x_{0}\right)$. Кроме того, вариационное неравенство (4.7) с помощью $\psi$ примет вид

$$
C_{1}\left(b^{*} \psi+r-v\right) \in N_{\mathbb{V}(y)}(v) \quad \text { или } \quad\left\langle C_{1}\left(v-b^{*} \psi-r\right), w-v\right\rangle \geqslant 0 \quad \forall w \in \mathbb{V}(y) .
$$

Функциональное включение и неравенство в (4.8) эквивалентны поточечному включению (4.3). С помощью (4.3) можно сделать дополнительные заключения об оптимальном возмущении. Рассмотрим это включение в $\mathbb{R}^{l}$ как соотношение с параметрами $\mathbf{r}=(b, \psi, r)$ :

$$
C_{1}\left(b^{\prime} \psi+r-v\right) \in N_{\mathbf{V}}(v) .
$$

Из [13, теорема 2F.6] заключаем, что

$$
\left|v\left(\mathbf{r}_{1}\right)-v\left(\mathbf{r}_{2}\right)\right|_{C_{1}} \leqslant\left|b_{1}^{\prime} \psi_{1}+r_{1}-b_{2}^{\prime} \psi_{2}-r_{2}\right|_{C_{1}} .
$$

Предположим дополнительно, что тройка $\mathbf{y}=(y, r, s) \in L_{\infty}^{m+l+n}$. Тогда функция $\psi(t)$ как решение дифференциального уравнения в (4.2) будет липшицевой по $t$. В случае, когда функции $b(t)$ и $r(t)$ оказываются липшицевыми, получаем неравенство $\left|v\left(\mathbf{r}_{1}\right)(t)-v\left(\mathbf{r}_{2}\right)\left(t^{\prime}\right)\right|_{C_{1}} \leqslant$ $\left|b^{\prime}(t) \psi(t)+r(t)-b^{\prime}\left(t^{\prime}\right) \psi\left(t^{\prime}\right)-r\left(t^{\prime}\right)\right|_{C_{1}} \leqslant \beta\left|t-t^{\prime}\right|$. Здесь $b_{1}=b(t), b_{2}=b\left(t^{\prime}\right), \psi_{1}=\psi(t), \psi_{2}=\psi\left(t^{\prime}\right)$ в (4.9). Значит, оптимальное возмущение будет липшицевым по $t$, поскольку произведение липшицевых функций является липшицевым. С другой стороны, если у $\in L_{\infty}^{m+l+n}$, то выполняется неравенство

$$
\left|v\left(\mathbf{y}_{1}\right)(t)-v\left(\mathbf{y}_{2}\right)(t)\right|_{C_{1}} \leqslant\left|b^{\prime}(t)\left(\psi\left(\mathbf{y}_{1}\right)(t)-\psi\left(\mathbf{y}_{2}\right)(t)\right)+r_{1}(t)-r_{2}(t)\right|_{C_{1}} \quad \text { почти всюду. }
$$

Здесь $b_{1}=b_{2}=b(t), r_{1}=r_{1}(t), r_{2}=r_{2}(t), \psi_{1}=\psi\left(\mathbf{y}_{1}\right)(t), \psi_{2}=\psi\left(\mathbf{y}_{2}\right)\left(t^{\prime}\right)$ в $(4.9)$. В теореме 2 уже установлено, что оптимальная траектория $x(t)$ является глобально липшицевым отображением по $(r, s)$ из $L_{2}^{l+n}$ в $\mathcal{C}^{n}$. Взяв простую оценку интегралов в неравенстве Липшица, убеждаемся, что траектория $x(t)$ будет глобально липшицевым отображением из $L_{\infty}^{l+n}$ в $\mathcal{C}^{n}$. Аналогичное можно утверждать и про отображение $\psi(t)$ в силу того, что эта функция является решением дифференциального уравнения в (4.2). Взяв норму в $L_{\infty}$ от левой и правой части неравенства (4.10) и произведя простые оценки, получаем, что оптимальное возмущение $v(\cdot)$ является глобально липшицевым отображением из $L_{\infty}^{l+n}$ в $L_{\infty}^{l}$. Справедлива теорема. 
Теорема 3. Пусть вектор-функции $b(t)$ u $r(t)$ являются липшицевыми по $t$ на рассматриваемом отрезке времени. Тогда оптимальное возмущение $v(t)$ из включения (4.1) также оказывается липшицевым по $t$. Отображение $(r, s) \mapsto(v, x, \psi)$ для оптимальной системы (4.2) лвляется глобально липшицевым из $L_{\infty}^{l+n}$ в пространство $L_{\infty}^{l} \times \mathcal{C}^{n} \times \mathcal{C}^{n}$ равномерно по всем сигналам у.

Рассмотрим теперь вопрос о том, как полученные результаты об оптимальном возмущении позволяют найти ИМ $\mathcal{X}_{T}(y)$. В общем случае, когда $y(\cdot) \in L_{2}^{m}$, получаем теорему.

Теорема 4. Задача построения ИМ $\mathcal{X}_{T}(y)$ при ограничениях (4.1) и при условиях (1.3) сводится $\kappa$ определению множества векторов $\left\{x_{T}\right\}$, для которых единственное решение $v(\cdot)$ оптимальной системы (4.2), (4.3) при подстановке в функционал $\psi_{X_{0}}\left(x_{0}\right)+J\left(T, x_{T}, v, y\right)$ дает значение, меньшее единицы.

\section{5. Соотношения для дискретной аппроксимации}

Отметим, что применение теоремы 4 для численного решения задачи построения ИМ $\mathcal{X}_{T}(y)$ достаточно затруднительно даже в случае гладких оптимальных возмущений. Примем для упрощения рассуждений, что функции $s(t)$ и $r(t)$ в функционалах $(1.2),(2.1)$ являются нулевыми, $y(\cdot) \in L_{\infty}^{m}$ и функция $b(t)$ оказывается липшицевой по $t$. В частности, так будет, если $b(t)=$ const. Разделим интервал $0 \leqslant t \leqslant T$ на $N$ частей $\left[t_{k-1}, t_{k}\right]$ одинаковой длины $\delta=t_{k}-t_{k-1}=1 / N$ точками $t_{k}, k \in 1: N$, с $t_{0}=0$ и $t_{N}=T$. Для аппроксимации оптимального возмущения, которое оказывается липшицевым по теореме 3 , используем кусочно-постоянные функции, сохраняющие постоянное значение $v(t)=v_{k}$ на полуинтервалах $\left(t_{k-1}, t_{k}\right]$ и на первом отрезке $\left[t_{0}, t_{1}\right]$, т. е. непрерывные слева. Они должны удовлетворять включениям $v_{k} \in \mathbf{V}$, и для всех таких функций функционал $J\left(T, x_{T}, v, y\right)$ должен быть равномерно ограничен сверху для фиксированного значения $x_{T}$. Будем решать первую систему в (4.2) справа налево, а вторую систему - слева направо, используя фундаментальную матрицу $\widetilde{X}(t, s)$ из $(2.5)$. Получаем

$$
\begin{gathered}
x_{k-1}=\tilde{X}\left(t_{k-1}, t_{k}\right) x_{k}-\int_{t_{k-1}}^{t_{k}} \tilde{X}\left(t_{k-1}, t\right)\left(\tilde{b}(t) y(t)+b(t) C_{1} v_{k}\right) d t, \quad x_{N}=x_{T}, \\
\psi_{k}=\tilde{X}^{\prime}\left(t_{k-1}, t_{k}\right) \psi_{k-1}-\int_{t_{k-1}}^{t_{k}} \tilde{X}^{\prime}\left(t, t_{k}\right) G^{\prime}(t) C\left(y(t)-G(t) x_{k}(t)\right) d t, \quad \psi_{0} \in P_{0} x_{0}+N_{X_{0}}\left(x_{0}\right), \\
C_{1}\left(b_{k}^{\prime} \psi_{k}-v_{k}\right) \in N_{\mathbf{V}}\left(v_{k}\right), \quad k \in 1: N,
\end{gathered}
$$

где $b_{k}=b\left(t_{k}\right)$. Включение (4.3) учитывается в (5.1) только в узловых точках $t_{k}$ разбиения. Функция $x_{k}(t)$ поясняется ниже в формуле (5.2).

Предположим, что систему (5.1) можно точно решить при любом $N$ и заданных краевых условиях. В результате решения получим последовательности векторов $\psi_{1: N}, x_{0: N}$ и $v_{1: N}$. Для данного $N$ эти последовательности идентифицируются с функциями

$$
\begin{gathered}
x_{k}(t)=\tilde{X}\left(t, t_{k}\right) x_{k}-\int_{t}^{t_{k}} \tilde{X}(t, s)\left(\tilde{b}(s) y(s)+b(s) C_{1} v_{k}\right) d s, \\
\psi_{k}(t)=\tilde{X}^{\prime}\left(t_{k-1}, t\right) \psi_{k-1}-\int_{t_{k-1}}^{t} \tilde{X}^{\prime}(s, t) G^{\prime}(s) C\left(y(s)-G(s) x_{k}(s)\right) d s, \\
t_{k-1} \leqslant t \leqslant t_{k}, \quad k \in 1: N .
\end{gathered}
$$


Обозначим непрерывные и кусочно-дифференцируемые вектор-функции, построенные на отрезке $0 \leqslant t \leqslant T$ из соотношений (5.2) путем склеивания, через $\tilde{x}(t)$ и $\tilde{\psi}(t)$. Пусть также $\tilde{v}(t)-$ кусочно-постоянная вектор-функция, для которой $\tilde{v}(t) \equiv v_{k}$ на интервале $\left(t_{k-1}, t_{k}\right]$.

Теорема 5. Рассмотрим задачу минимизации функиионала (4.1) с условиями (1.3) и нулевыми функииями $(s(\cdot), r(\cdot))$. Сигнал считаем существенно ограниченной функиией. Пусть решение оптимальной системь (4.2), (4.3) с липшицевой функцией $v(t)$ nо $t$ образует набор $(v(t), x(t), \psi(t))$. Рассмотрим также векторную дискретизацию $(\tilde{v}(t), \tilde{x}(t), \tilde{\psi}(t))$, полученную из соотношений (5.2). Тогда имеет место следующая ощенка

$$
\left\|C_{1}(\tilde{v}(\cdot)-v(\cdot))\right\|_{\infty}+\|\tilde{x}(\cdot)-x(\cdot)\|_{\mathcal{C}}+\|\tilde{\psi}(\cdot)-\psi(\cdot)\|_{\mathcal{C}} \leqslant \text { const } \cdot \delta
$$

т.е. левая часть неравенства (5.3) имеет порядок малости $O(\delta)$.

Д о к а з а т е л ь с т в о. Для почти всех $t \in\left(t_{k-1}, t_{k}\right]$ будем иметь после дифференцирования функций в (5.2) соотношения

$$
\begin{gathered}
\dot{\tilde{x}}(t)=\widetilde{A}(t) \tilde{x}(t)+\tilde{b}(t) y(t)+b(t) C_{1} \tilde{v}(t), \quad \tilde{x}\left(t_{k}\right)=x_{k}, \\
\dot{\tilde{\psi}}(t)=-\widetilde{A}^{\prime}(t) \tilde{\psi}(t)-G^{\prime}(t) C(y(t)-G(t) \tilde{x}(t)), \quad \tilde{\psi}\left(t_{k}\right)=\psi_{k}, \quad \tilde{\psi}_{0} \in P_{0} x_{0}+N_{X_{0}}\left(x_{0}\right), \\
C_{1}\left(b^{\prime}(t) \tilde{\psi}(t)+r^{N}(t)-\tilde{v}(t)\right) \in N_{\mathbf{V}}(\tilde{v}(t)),
\end{gathered}
$$

где $r^{N}(t)=b_{k}^{\prime} \psi_{k}-b^{\prime}(t) \tilde{\psi}(t)$ на интервале $\left(t_{k-1}, t_{k}\right]$, причем $r^{N}\left(t_{k}\right)=0, k \in 1: N$. Функция $r^{N}(t)$ кусочно-непрерывна, непрерывна слева и ограничена. Действительно, установим ограниченность. В силу неравенства (2.1) и фиксированного состояния $x(T)=x_{T}$ все начальные состояния $x_{0}$ ограничены по норме и все возможные функции $v(\cdot)$ ограничены в пространстве $L_{2}^{l}$. Отсюда оптимальная пара $(x(\cdot), \psi(\cdot))$ из пространства $\mathcal{C}^{2 n}$ содержится в шаре этого пространства с радиусом, зависящим от нормы $\|y(\cdot)\|_{\infty}$ сигнала. Значит, и последовательности векторов $\psi_{1: N}, x_{0: N}$, а также и $C_{1} v_{1: N}$ ограничены. Система (5.4) имеет такой же вид, как (4.1), (4.2) при специальном выборе параметров. Согласно теореме 3 для оптимальной тройки $(v(t), x(t), \psi(t))$ с нулевым набором $(s(\cdot), r(\cdot))$ и тройки $(\tilde{v}(t), \tilde{x}(t), \tilde{\psi}(t))$ получаем неравенство

$$
\left\|C_{1}(\tilde{v}(\cdot)-v(\cdot))\right\|_{\infty}+\|\tilde{x}(\cdot)-x(\cdot)\|_{\mathcal{C}}+\|\tilde{\psi}(\cdot)-\psi(\cdot)\|_{\mathcal{C}} \leqslant \text { const } \cdot\left\|r^{N}(\cdot)\right\|_{\infty} .
$$

Покажем, что величина справа в неравенстве (5.5) имеет порядок $O(\delta)$. Фундаментальные матрицы $\tilde{X}(t, s)$ и $X(t, s)$ оцениваются одинаково по лемме Гронуолла

$$
\|X(t, s)\| \leqslant \exp (a|t-s|), \quad\left\|X(t, s)-I_{n}\right\| \leqslant a|t-s| \exp (a|t-s|),
$$

где $\|\cdot\|$ - евклидова норма матрицы, $a$ - константа. Поскольку $\left|r^{N}(t)\right| \leqslant\left|b_{k}^{\prime}\right|\left|\psi_{k}-\tilde{\psi}(t)\right|+\mid b_{k}^{\prime}-$ $b^{\prime}(t)|| \tilde{\psi}(t) \mid$ на каждом отрезке $\left[t_{k-1}, t_{k}\right]$, то в силу ограниченности соответствующих величин и липшицевости функций $b(t), \tilde{\psi}(t)$ порядок $O(\delta)$ для $r^{N}(t)$ установлен.

Отметим, что в теореме 5 существенно использован тот факт, что система вида (4.2), (4.3) имеет единственное решение. Оценка (5.3) в теореме 5 справедлива и для функционалов с $(r, s) \neq 0$.

3 а м е ч а н и е 2. Предположим, что имеют место соотношения (2.6)-(2.8). Тогда слагаемое $\left\|C_{1}(\tilde{v}(\cdot)-v(\cdot))\right\|_{\infty}$ в оценке $(5.3)$ заменяется на $\left.\| \tilde{w}(\cdot)-w(\cdot)\right) \|_{\infty}$. Отметим также, что вместо (5.2) будем иметь

$$
\begin{gathered}
x_{k}(t)=X\left(t, t_{k}\right) x_{k}-\int_{t}^{t_{k}} X(t, s) b(s) w_{k} d s, \quad t_{k-1} \leqslant t \leqslant t_{k}, \quad k \in 1: N, \\
\psi_{k}(t)=X^{\prime}\left(t_{k-1}, t\right) \psi_{k-1}-\int_{t_{k-1}}^{t} X^{\prime}(s, t) G^{\prime}(s)\left(y(s)-G(s) x_{k}(s)\right) d s .
\end{gathered}
$$


3 а м е ч а н и е 3. Если множество допустимых значений векторов $x_{T}$ в формулах (4.2), (5.1)-(5.4) является ограниченным по условию, то величина const в оценке (5.3) зависит только от $y(\cdot)$ и не зависит от $x_{T}$, т. е. оценка (5.3) является равномерной по всем допустимым $x_{T}$. Данный факт будет справедливым, если $P_{0}>0$ или $X_{0}$ является компактом.

\section{1. О решении дискретной системы}

Остановимся на способах решения системы (5.1). Эта система соответствует задаче оптимального управления, состоящей в минимизации функционала

$$
J^{N}\left(x_{T}, v_{1: N}\right)=\psi_{X_{0}}\left(x_{0}\right)+\left|x_{0}\right|_{P_{0}}^{2}+\sum_{k=1}^{N} \int_{t_{k-1}}^{t_{k}}\left(\left|y(t)-G(t) x_{k}(t)\right|_{C}^{2}+\left|v_{k}\right|_{C_{1}}^{2}\right) d t, \quad v_{k} \in \mathbf{V},
$$

на решениях первой дискретной системы в (5.1). Здесь функция $x_{k}(t)$ определена в (5.2). Представим систему как

$$
\begin{gathered}
x_{k-1}=\bar{A}_{k} x_{k}+\bar{y}_{k}+\bar{b}_{k} C_{1} v_{k}, \quad k \in 1: N, \quad x_{N}=x_{T}, \quad \text { где } \\
\bar{A}_{k}=\tilde{X}\left(t_{k-1}, t_{k}\right), \quad \bar{y}_{k}=-\int_{t_{k-1}}^{t_{k}} \tilde{X}\left(t_{k-1}, t\right) \tilde{b}(t) y(t) d t, \quad \bar{b}_{k}=-\int_{t_{k-1}}^{t_{k}} \tilde{X}\left(t_{k-1}, t\right) b(t) d t .
\end{gathered}
$$

Функционал (5.6) перепишем как

$$
J^{N}\left(x_{T}, v_{1: N}\right)=\psi_{X_{0}}\left(x_{0}\right)+\left|x_{0}\right|_{P_{0}}^{2}+\sum_{k=1}^{N} F_{k}\left(x_{k}, v_{k}\right), \quad v_{k} \in \mathbf{V} .
$$

Дискретная задача является линейно-выпуклой, и поэтому ее можно решить с помощью дискретного принципа максимума (см. [14]). Для рассматриваемой задачи этот принцип, являющийся необходимым и достаточным условием, выглядит следующим образом. Найдутся сопряженные переменные: число $\psi^{0}>0$ и последовательность векторов $\psi_{0: N}$ такие, что выполняются уравнения

$$
\begin{aligned}
\psi_{0} \in 2 \psi^{0} P_{0} x_{0}+ & N_{X_{0}}\left(x_{0}\right), \quad \psi_{k}=\psi_{0} \nabla_{x} F_{k}\left(x_{k}, v_{k}\right)+\bar{A}_{k}^{\prime} \psi_{k-1}, \quad k \in 1: N, \\
& -\psi^{0} \nabla_{v} F_{k}\left(x_{k}, v_{k}\right)-C_{1} \bar{b}_{k}^{\prime} \psi_{k-1} \in N_{\mathbf{V}}\left(v_{k}\right) .
\end{aligned}
$$

Здесь $\nabla_{x} F(x)$ - матрица Якоби для $m$-векторной функции $F(x)$ от $n$ переменных, равная $\left[\nabla_{x} F^{1}(x), \ldots, \nabla_{x} F^{m}(x)\right]$. Для скалярной функции это градиент (вектор-столбец). Параметры для (5.9) определены в (5.7), (5.8). Включение в (5.9) следует из [13, теорема А.7]. Полагаем $\psi^{0}=1 / 2$.

Вычисляя производные в (5.9), приходим к дискретной системе (5.2) и включению

$$
\begin{gathered}
C_{1}\left(\int_{t_{k-1}}^{t_{k}} \bar{b}_{k}^{\prime}(t) G^{\prime}(t) C\left(y(t)-G(t) x_{k}(t)\right) d t-v_{k} \delta-\bar{b}_{k}^{\prime} \psi_{k-1}\right) \in N_{\mathbf{V}}\left(v_{k}\right), \\
\bar{b}_{k}(t)=-\int_{t}^{t_{k}} \widetilde{X}(t, s) b(s) d s .
\end{gathered}
$$

Полученное включение (5.10) в (5.9) несколько отличается от включения в (5.1). Однако это отличие несущественно. В силу однородности можем разделить правую и левую часть включения (5.10) на $\delta$. Пользуясь липшицевостью функции $b(t)$, получаем оценки

$$
\left|\bar{b}_{k} / \delta+\widetilde{X}\left(t_{k-1}, t_{k}\right) b_{k}\right| \leqslant \text { const } \cdot \delta, \quad\left|\bar{b}_{k}(t) / \delta+\widetilde{X}\left(t, t_{k}\right) b_{k}\right| \leqslant \text { const } \cdot \delta .
$$


Отсюда и из второго уравнения в (5.1) следует, что правая часть включения в (5.10), деленная на $\delta$, отличается от величины $C_{1}\left(b_{k}^{\prime} \psi_{k}-v_{k}\right)$ на порядок $O(\delta)$, что в комбинации с результатом теоремы 5 подтверждает оценку (5.3).

\section{2. Аппроксимация информационных множеств}

Предположим, что для некоторого состояния $x_{T}$ данного сигнала $y(\cdot)$ и некоторого разбиения отрезка $0 \leqslant t \leqslant T$ значение минимума функционала $J^{N}\left(x_{T}, v_{1: N}\right)$ из (5.6) меньше единицы. Введем ИМ $\mathcal{X}_{T}^{N}(y)$ дискретной задачи по формуле $\mathcal{X}_{T}^{N}(y)=\left\{x_{T} \in \mathbb{R}^{n} \mid J^{N}\left(x_{T}, v_{1: N}\right)<1\right\}$. Это множество будет непустым по предположению. Поскольку минимум функционала в (4.1) заведомо меньше, чем минимум в (5.6), имеем включение

$$
\mathcal{X}_{T}^{N}(y) \subset \mathcal{X}_{T}(y) \quad \forall N .
$$

Отметим, что в силу наложенных ограничений множества во включении (5.11) являются ограниченными. Поэтому можем рассмотреть хаусдорфово расстояние между ними

$$
h\left(\mathcal{X}_{T}^{N}(y), \mathcal{X}_{T}(y)\right)=\max \left\{\sup _{a \in \mathcal{X}_{T}^{N}(y)} \inf _{b \in \mathcal{X}_{T}(y)}|a-b|, \sup _{b \in \mathcal{X}_{T}(y)} \inf _{a \in \mathcal{X}_{T}^{N}(y)}|a-b|\right\} .
$$

Ввиду включения (5.11) максимум здесь всегда достигается на втором выражении. При увеличении номера $N$ аппроксимационные множества монотонно расширяются

$$
\mathcal{X}_{T}^{1}(y) \subset \mathcal{X}_{T}^{2}(y) \subset \ldots,
$$

поскольку множество, по которому производится минимизация функционала (5.6), расширяется с ростом $N$. Начиная с некоторого номера, все ИМ во включениях (5.12) становятся непустыми. Следовательно, хаусдорфово расстояние $h\left(\mathcal{X}_{T}^{N}(y), \mathcal{X}_{T}(y)\right)$ монотонно убывает.

В случае равномерной по $x_{T}$ оценки (5.3), учитывая липшицевость функционалов (4.1) и (5.6) по параметрам, получаем оценку

$$
\left|\psi_{X_{0}}\left(x_{0}\right)+J\left(T, x_{T}, v, y\right)-J^{N}\left(x_{T}, v_{1: N}\right)\right| \leqslant \text { const } \cdot \delta
$$

равномерную по допустимым векторам $x_{T}$. Следовательно, $h\left(\mathcal{X}_{T}^{N}(y), \mathcal{X}_{T}(y)\right) \rightarrow 0$ при $N \rightarrow \infty$. Сформулируем теорему.

Теорема 6. Предположим, что $P_{0}>0$ или множество $X_{0}$ является компактом. Тогда при условиях теоремы 5 хаусдорфово расстояние $h\left(\mathcal{X}_{T}^{N}(y), \mathcal{X}_{T}(y)\right)$ между аппроксимирующим ИМ и исходным ИМ монотонно стремится к нулю при $N \rightarrow \infty$.

\section{6. Случай с ограничением на начальное состояние и интегральными ограничениями на возмущения}

Предположим, что имеется ограничение $x_{0} \in X_{0}$, где $X_{0}$ - выпуклое замкнутое множество, содержащее начало координат, и $P_{0}=0$. Пусть также $\mathbf{V}=\mathbb{R}^{l}$ и выполняются условия (1.3). Если $X_{0}$ компактно, выберем невырожденную матрицу $Z^{\prime}=Z>0$ так, чтобы

$$
\left\{\left.x|| x\right|_{Z} ^{2}<1\right\} \supset X_{0}
$$

и рассмотрим ограничение

$$
(1-\alpha)\left|x_{0}\right|_{Z}^{2}+\alpha J\left(T, x_{T}, v, y\right)<1
$$


где $\alpha \in(0,1)$. Обозначим через $\mathcal{X}_{T}^{Z, \alpha}(y)$ ИМ, построенное при ограничениях $(6.1),(6.2)$. Исходное ИМ $\mathcal{X}_{T}(y)$, которое, вообще говоря, не является эллипсоидом, будет удовлетворять включению

$$
\mathcal{X}_{T}(y) \subset \mathcal{X}_{T}^{Z, \alpha}(y)
$$

при любых допустимых параметрах $P_{0}, \alpha$. Следовательно, $\mathcal{X}_{T}(y) \subset \bigcap_{Z, \alpha} \mathcal{X}_{T}^{P_{0}, \alpha}(y)$.

Пусть $P_{T}^{Z, \alpha}$ - матрица эллипсоида $\mathcal{X}_{T}^{Z, \alpha}(y)$, а величина $h_{T}^{Z, \alpha}$ означает соответствующее значение $h$ в формуле (3.10). Поскольку объем невырожденного эллипсоида $\left\{x \mid x^{\prime} P x<1\right\}$ пропорционален величине $(\operatorname{det} P)^{-1 / 2}($ см. [5]), где $\operatorname{det} P-$ определитель матрицы эллипсоида, то имеет смысл выбирать параметры $Z, \alpha$ как результат решения экстремальной задачи

$$
Q(Z, \alpha, y)=\operatorname{det} P_{T}^{Z, \alpha} /\left(1-h_{T}^{Z, \alpha}\right) \rightarrow \sup _{Z, \alpha}
$$

с целью минимизации объема аппроксимирующего эллипсоида в (6.3). Многие задачи минимизации объема аппроксимирующих эллипсоидов исследовались в монографиях [5-7]. Однако точно такой, как (6.4), среди них нет. Численное решение этой задачи является достаточно сложным.

Можно указать случай, когда в решении задачи (6.4) нет необходимости. Пусть система (1.1) имеет вид

$$
\dot{x}(t)=A(t) x(t), \quad y(t)=G(t) x(t)+v(t), \quad 0 \leqslant t \leqslant T, \quad x_{0} \in X_{0},
$$

где функция $v(\cdot)$ интегрально ограничена: $\int_{0}^{T}|v(t)|^{2} d t<\mu^{2}$. Предполагая, что выполнены условия невырожденности (3.7), по формулам (3.3), (3.9) строим невырожденный эллипсоид $\mathcal{X}_{T}^{0}(y)$ без ограничений на начальное состояние. Тогда точное ИМ выразится формулой

$$
\mathcal{X}_{T}(y)=\mathcal{X}_{T}^{0}(y) \bigcap X(T, 0) X_{0},
$$

где $X(T, 0)$ - фундаментальная матрица и $X_{0}$ - начальное множество. В общем случае системы (1.1) точное ИМ строится приближенно.

Рассмотрим пример.

П р и м е р. Рассмотрим движение материальной точки по прямой, подверженное возмущению $v^{1}(t)$ :

$$
\dot{x}^{1}=x^{2}, \quad \dot{x}^{2}=v^{1}(t), \quad 0 \leqslant t \leqslant T .
$$

Пусть возмущение $v^{1}$ влияет также и на уравнение наблюдения, где дополнительно присутствует шум измерения $v^{2}$ :

$$
y(t)=x^{1}(t)+v^{1}(t)+v^{2}(t) .
$$

Данные уравнения приводят к системе вида (2.5)

$$
\dot{x}^{1}=x^{2}, \quad \dot{x}^{2}=\left(y-x^{1}+v^{1}-v^{2}\right) / 2 .
$$

Вектор-функция $v(t)$ стеснена здесь интегральным ограничением (1.2), где $r=0, s=0, \mathbf{V}=\mathbb{R}^{2}$ и $X_{0}=\left\{x \in \mathbb{R}^{2}|| x_{0}^{1}|\leqslant 1,| x_{0}^{2} \mid \leqslant 1\right\}$, т. е. имеем случай отсутствия геометрических ограничений на $v(t)$. Функционал из (2.1) принимает вид

$$
J\left(T, x_{T}, v, y\right)=\int_{0}^{T}\left(\left|y(t)-x^{1}(t)\right|^{2}+\left|v^{1}(t)-v^{2}(t)\right|^{2}\right) d t / 2 .
$$




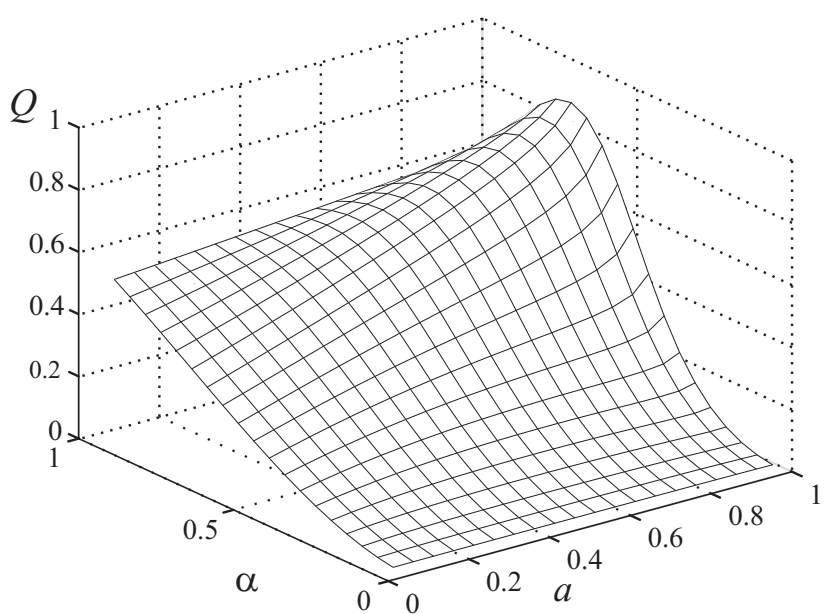

График функции $Q$

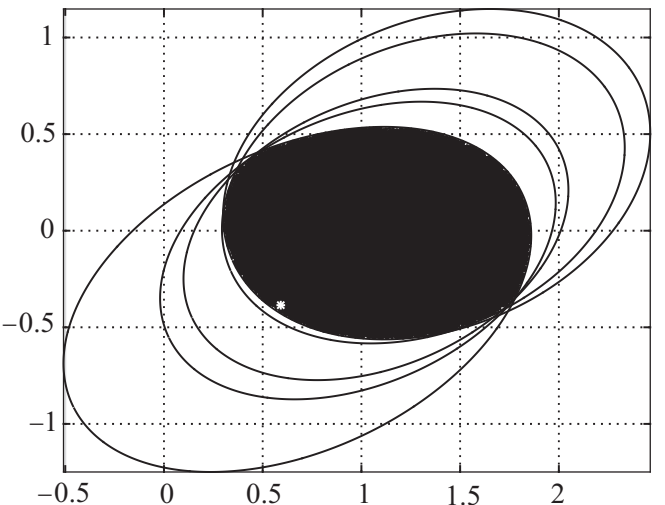

Пересечение эллипсов-ИМ

Возьмем в качестве ограничения на начальные состояния однопараметрическое семейство эллипсов с диагональной матрицей $P_{0}=[a, 0 ; 0,1-a]$, где $a \in(0,1)$. Тогда любой эллипс $\left\{x \mid x^{\prime} P_{0} x \leqslant 1\right\}$ будет содержать квадрат начальных состояний $X_{0}$. Выберем еще один параметр $\alpha \in(0,1)$ и рассмотрим ограничение

$$
(1-\alpha)\left|x_{0}\right|_{P_{0}}^{2}+\alpha J\left(T, x_{T}, v, y\right)<1,
$$

где функционал $J$ определен в (6.6). ИМ $\mathcal{X}_{T}^{a, \alpha}(y)$ для системы (6.5) с ограничением (6.7) будет содержать исходное ИМ $\mathcal{X}_{T}(y)$ при всяком сигнале в исходной системе. Воспользуемся соотношениями (3.3), (3.10), (3.11). Имеем

$$
\begin{gathered}
\mathcal{X}_{T}^{a, \alpha}(y)=\left\{x_{T}|| x_{T}-\left.\hat{x}(T)\right|_{P(T)} ^{2}+h(T)<1\right\}, \\
\dot{P}=-P(t) \widetilde{A}-\widetilde{A}^{\prime} P(t)+\alpha G^{\prime} G / 2-P(t) b C_{1} b^{\prime} P(t) / \alpha, \quad P(0)=(1-\alpha) P_{0} ; \\
\dot{\hat{x}}(t)=A \hat{x}(t)+\alpha\left(b c^{\prime}+P^{-1}(t) G^{\prime}\right)\left(y(t)-\hat{x}^{1}(t)\right) / 2, \quad \dot{h}(t)=\alpha\left|y(t)-\hat{x}^{1}(t)\right|^{2} / 2 .
\end{gathered}
$$

Здесь параметры имеют вид $b=[0,0 ; 1,0], c=[1,1], G=[1,0], C=1 / 2, C_{1}=[1,-1 ;-1,1] / 2$, $\widetilde{A}=[0,1 ;-1,0]$.

Для иллюстрации выберем некоторый сигнал, порожденный допустимыми функциями $v^{1}(t)=0.8 \cos (t), v^{2}(t)=0.8 \sin (t)$ и допустимым начальным состоянием $x_{0}=[1 ; 1] / 2$. Функция $Q$ из (6.4) будет зависеть от двух параметров при фиксированном сигнале $y$. График этой функции и пересечение пяти эллипсов в момент $T=3$ при разных параметрах показаны на рисунках. Темное пятно - это аппроксимация сверху для точного ИМ. Светлая звездочка означает истинное состояние, принадлежащее всем эллипсам-ИМ. Особенность задачи максимизации (6.4) здесь состоит в том, максимум по $P_{0}$ достигается на вырожденной матрице, т. е. при $a=1$. В связи с этим были использованы соотношения (3.5), (3.9). Величина $\max Q \approx 0.8696$. Точное значение ИМ для этого примера построить не удается. Приближенному построению ИМ для этого и других примеров будет посвящена отдельная работа.

\section{Заключение}

1. Рассмотрена конечномерная аппроксимация задачи гарантированного оценивания для линейных нестационарных систем с возмущениями, подчиненными смешанным интегральным и геометрическим ограничениям.

2. При условии, что параметры системы и уравнения измерения формируются без геометрических ограничений на фазовый вектор системы, задача оценивания сведена к задаче 
оптимального управления без фазовых ограничений, которая решается с помощью принципа максимума Л. С. Понтрягина или его обобщения.

3. Указана дискретная многошаговая система, для которой информационное множество сходится в метрике Хаусдорфа к соответствующему информационному множеству непрерывной системы при измельчении разбиения отрезка наблюдения. Получены оценки, характеризующие скорость сходимости.

4. Рассмотрен численный пример аппроксимации ИМ при раздельных геометрических ограничениях на начальное состояние и интегральных ограничениях на помехи.

\section{СПИСОК ЛИТЕРАТУРЫ}

1. Куржанский А.Б. Управление и наблюдение в условиях неопределенности. М.: Наука, 1977. $306 \mathrm{c.}$

2. Bertsecas D.P., Rhodes I. B. Recursive state estimation for a set-memberschip description of uncertainty // IEEE Trans. on Auto. Control. 1971. AC-16, no. 2. P. 117-128. doi: 10.1109/TAC.1971.1099674.

3. Schweppe F. C. Uncertain dynamic systems. N Y: Prentice Hall, 1973. 563 p.

4. Schmitendorf W. E. Minimax control of systems with uncertainty in the initial state and in the state equations // IEEE Trans. on Auto. Control. 1971. AC-22, no. 3. P. 439-443. doi: 10.1109/TAC.1977.1101506 .

5. Черноусько Ф. Л. Оценивание фазового состояния динамических систем. Метод эллипсоидов. М.: Наука, 1988. 319 с.

6. Kurzhanski A. B., Valyi I. Ellipsoidal calculus for estimation and control. Boston: Birkhäuser, 1996. $321 \mathrm{p}$.

7. Kurzhanski A. B., Varaiya P. Dynamics and control of trajectory tubes: Theory and computation. SCFA, Birkhäuser, 2014. 445 p.

8. Ananyev B.I., Yurovskih P. A. On the approximation of estimation problems for controlled systems // AIP Conf. Proc. 2019. Vol. 2164, iss. 1. Art.-no. 110001. 1-9 p. doi: 10.1063/1.5130846.

9. Ананьев Б. И. Минимаксная квадратичная задача коррекции движения // Прикладная математика и механика. 1977. Т. 41, № 3. С. 436-445.

10. Ананьев Б. И. Задача коррекции движения с гауссовским каналом связи // Автоматика и телемеханика. 2011. № 2. С. 25-40.

11. Васильев Ф. П. Численные методы решения экстремальных задач. М.: Наука, 1988. 552 с.

12. Кларк Ф. Оптимизация и негладкий анализ. М.: Наука, 1988. 280 с.

13. Dontchev A., Rockafellar T. Implicit functions and solution mappings. A view from variational analysis. N Y: Springer, 2014. 466 p.

14. Пропой А. И. Элементы теории оптимальных дискретных процессов. М.: Наука, 1973. 256 с.

Поступила 30.08.2020

После доработки 19.10.2020

Принята к публикации 26.10.2020

Ананьев Борис Иванович

д-р физ.-мат. наук

ведущий науч. сотрудник

Институт математики и механики им. Н. Н. Красовского УрО РАН

г. Екатеринбург

e-mail: abi@imm.uran.ru

Юровских Полина Александровна

аспирант

Институт математики и механики им. Н. Н. Красовского УрО РАН

г. Екатеринбург

e-mail: polina2104@list.ru 


\section{REFERENCES}

1. Kurzhanskii A.B. Upravlenie $i$ nablyudenie $v$ usloviyakh neopredelennosti [Control and Observation Under the Conditions of Uncertainty]. Moscow: Nauka Publ., 1977, 306 p.

2. Bertsecas D.P., Rhodes I.B. Recursive state estimation for a set-membership description of uncertainty. IEEE Trans. on Auto. Control, 1971, vol. 16, no. 2, pp. 117-128. doi: 10.1109/TAC.1971.1099674.

3. Schweppe F.C. Uncertain dynamic systems. N Y: Prentice Hall, 1973, 563 p. ISBN: 0139355936.

4. Schmitendorf W.E. Minimax Control of Systems with Uncertainty in the Initial State and in the State Equations. IEEE Trans. on Auto. Control, 1971, vol. 22, no. 3, pp. 439-443. doi: 10.1109/TAC.1977.1101506.

5. Chernous'ko F.L. Otsenivanie fazovogo sostoyaniya dinamicheskikh sistem. Metod ellipsoidov. (Phase state estimation for dynamical systems. The method of ellipsoids.) Moscow: Nauka Publ., 1988, 319 p. ISBN: 5-02-013899-1.

6. Kurzhanski A.B., Valyi I. Ellipsoidal Calculus for Estimation and Control. Basel: Birkhäuser, 1997, 321 p. ISBN: 978-0-8176-3699-9 .

7. Kurzhanski A.B., Varaiya P. Dynamics and Control of Trajectory Tubes: Theory and Computation. SCFA, vol. 85. Basel: Birkhäuser, 2014, 445 p. doi: 10.1007/978-3-319-10277-1.

8. Ananyev B.I., Yurovskih P.A. On the Approximation of Estimation Problems for Controlled Systems. AIP Conference Proceedings, 2019, vol. 2164, iss. 1, art.-no. 110001, 9 p. doi: 10.1063/1.5130846.

9. Anan'ev B.I. Minimax quadratic problem of motion correction. J. Appl. Math. Mech., 1977, vol. 41, no. 3, pp. 434-445. doi: 10.1016/0021-8928(77)90035-1.

10. Anan'ev B.I. The problem of motion correction with Gaussian communications channel. Autom. Remote Control, 2011, vol. 72, no. 2, pp. 240-254. doi: 10.1134/S0005117911020044.

11. Vasilyev F.P. Chislennye metody resheniya ekstremal'nykh zadach [Numerical methods for solving extremal problems]. Moscow: Nauka Publ., 1988, 552 p. ISBN: 5-02-013796-0 .

12. Clarke H. Optimization and nonsmooth analysis. Canadian Mathematical Society Series in Mathematics, N Y et al., John Wiley \& Sons, 1983, 308 p.

13. Dontchev A., Rockafellar T. Implicit functions and solution mappings. A view from variational analysis, N Y: Springer, 2014, $466 \mathrm{p}$.

14. Propoy A.I. Elementy teorii optimal'nykh diskretnykh protsessov (Elements of the theory of optimal discrete processes). Moscow: Nauka Publ., 1973, 256 p.

Received August 30, 2020

Revised October 19, 2020

Accepted October 26, 2020

Funding Agency: This study was supported by the Scientific Educational Center of the IMM UB RAS working under the Ural Mathematical Center.

Boris Ivanovich Ananyev, Dr. Phys.-Math. Sci., Leader Sci. Collaborator, Krasovskii Institute of Mathematics and Mechanics of the Ural Branch of the Russian Academy of Sciences, Yekaterinburg, 620108 Russia, e-mail: abi@imm.uran.ru .

Polina Aleksandrovna Yurovskih, doctoral student, Krasovskii Institute of Mathematics and Mechanics of the Ural Branch of the Russian Academy of Sciences, Yekaterinburg, 620108 Russia, e-mail: polina2104@list.ru .

Cite this article as: B.I. Anan'ev, P. A. Yurovskikh. Approximation of a guaranteed estimation problem with mixed constraints, Trudy Instituta Matematiki i Mekhaniki UrO RAN, 2020, vol. 26, no. 4 , pp. $48-63$. 